\title{
Mappemonde
}

Revue trimestrielle sur l'image géographique et les formes du territoire

129 | 2020

Varia

\section{Impression, soleil levant de Monet : le port du Havre comme porte d'entrée picturale dans l'Anthropocène?}

\section{Michel Rasse}

\section{(2) OpenEdition}

1 Journals

\section{Édition électronique}

URL : http://journals.openedition.org/mappemonde/4462

DOI : 10.4000/mappemonde.4462

ISSN : 1769-7298

Éditeur

UMR ESPACE

\section{Référence électronique}

Michel Rasse, «Impression, soleil levant de Monet : le port du Havre comme porte d'entrée picturale dans l'Anthropocène? », Mappemonde [En ligne], 129 | 2020, mis en ligne le 10 novembre 2020, consulté le 10 décembre 2020. URL : http://journals.openedition.org/mappemonde/4462 ; DOI : https://doi.org/10.4000/mappemonde.4462

Ce document a été généré automatiquement le 10 décembre 2020.

\section{(i) (2)}

La revue Mappemonde est mise à disposition selon les termes de la Licence Creative Commons Attribution - Pas d'Utilisation Commerciale - Partage dans les Mêmes Conditions 4.0 International. 


\title{
Impression, soleil levant de Monet : le port du Havre comme porte d'entrée picturale dans l'Anthropocène?
}

\author{
Michel Rasse
}

À la mémoire de Jean-Jacques Dufaure (1932-2020) ${ }^{1}$

Il est assez classique de revisiter le passé à la lumière des préoccupations actuelles et, à la faveur de la montée en puissance des interrogations environnementales, mais également à la lueur des recherches portant sur les conditions du développement économique et industriel $\mathrm{du} \mathrm{XIX}^{\mathrm{e}}$ siècle, bon nombre d'auteurs tentent de relire certaines récurrences picturales en prenant en considération de multiples aspects de l'histoire environnementale jusque-là délaissés. Ainsi Metzger A. et Tabeaud M. (2010) relisent les scènes hivernales du Siècle d'or hollandais à la faveur des études paléoclimatiques sur le Petit Âge glaciaire (même s'ils nuancent le propos en relativisant le strict rôle des conditions hivernales), Zerefos C. et al. (2014) et d'Arcy Wood G. (2019) font le parallèle entre la grande éruption du Tambora (Indonésie) en 1815 et les ciels tourmentés de Turner, et on sait le lien évoqué depuis toujours entre le brouillard sur la Tamise et la série consacrée par Monet au Parlement de Londres (Thornes et Metherell, 2003; l'exposition en 1904 des «Vues de la Tamise à Londres 1900-1904 » constitua un grand moment de la carrière du peintre). Aussi, pour paraphraser Dombrowski A. (Alphant et al., 2011), le paysage impressionniste «peut-il aujourd'hui nous inviter à réexaminer nos existences » et peut-il être lui-même réexaminé à la lumière d'une " relecture environnementale » de la deuxième moitié du $\mathrm{XIX}^{\mathrm{e}}$ siècle ? Les préoccupations environnementales actuelles laissent supposer que nos prédécesseurs n'étaient pas ou peu conscients des impacts de la révolution industrielle sur les paysages, sur les écosystèmes, sur l'atmosphère et l'hydrosphère. Or, comme le montrent très bien Bonneuil C. et Fressoz J.-B. dans L'événement Anthropocène. La Terre, 
l'histoire et nous (2016), de nombreux érudits ont très tôt tiré la sonnette d'alarme et on peut émettre l'idée que tout citoyen ait pu s'interroger sur les modifications environnementales subies durant son existence, au même titre que chacun d'entre nous s'interroge aujourd'hui sur le devenir d'une planète que l'on sait désormais «finie».

2 L'impressionnisme a été étudié sous tous les angles possibles. «L'époque, le milieu, le déclin de la peinture d'histoire, l'hédonisme, le photographique, le prosaïsme, la sublimation, la France républicaine, la légèreté transportable des tubes de couleurs, l'œil, la vitesse de la pochade et des échanges, la nouvelle organisation du marché de l'art, la civilisation des loisirs, la lumière, la naissance du cinématographe, l'intériconicité ${ }^{2}$ " (Alphant M. in Alphant et al., 2011) ont été successivement évoqués pour expliquer l'emprise, le pouvoir de ces images si connues de tous, si regardées qu'elles en deviennent presque lassantes tant elles sont démultipliées par les moyens modernes de reproduction. Dans toutes ces approches, rares sont celles qui relèvent de la géographie des paysages et des environnements. Rares aussi sont les études sur la perception des artistes des modifications environnementales contemporaines. Et si, après « la peinture d'histoire » (dont l'importance s'estompe beaucoup durant la fin du $\mathrm{XVIII}^{\mathrm{e}}$ et au début du XIX ${ }^{\mathrm{e}}$ siècle), le paysage impressionniste (voire tout le courant " impressionniste" dans son ensemble) était une "peinture de géographie »? Au simple sens d'une géographie des modifications majeures que le XIX ${ }^{\mathrm{e}}$ siècle a induites : essor industriel et urbain, exode rural et abandon de terroirs surexploités, développement des transports, affirmation de la bourgeoisie, nouveaux rapports sociaux, nouvelles distractions, etc. Les impressionnistes auraient-ils été sensibles à la sublimation des paysages, comme le furent en un temps les géographes, mais aussi à leur dégradation durant la deuxième moitié du XIX ${ }^{e}$ siècle, comme le sont aujourd'hui les environnementalistes, écologistes et autres défenseurs de la nature?

3 À ce titre, il nous paraît assez significatif de relever à la fois que la première loi de protection des oiseaux en Angleterre date de 1869 (la "Seabirds Protection Act»; en même temps que les premières législations sur la réduction de la pollution de l'air et de l'eau) et qu'en France, la Société pour la Protection des Paysages (devenue en 1954 Société pour la Protection des Paysages et de l'Esthétique de la France), plus ancienne association nationale pour la défense du patrimoine, ait été fondée en 1901, au tournant $\mathrm{du} \mathrm{XX}^{\mathrm{e}}$ siècle, soit après environ un demi-siècle de transformations importantes, tant industrielles, urbaines, sociétales, qu'environnementales. Dans le même ordre d'idée, la préservation par décret impérial en 1861 des 1097 hectares de la « série artistique » de la forêt de Fontainebleau, protégés de tout aménagement, sous l'impulsion du " peintre de Barbizon» Théodore Rousseau (proche du Duc de Morny, demi-frère de Napoléon III), ne constitue-t-elle pas la première mesure de protection environnementale au monde (et avant les protections américaines de Yosemite et de Yellowstone) ? En économie, Mill J. S., en 1857, est le premier à souligner les limites du modèle capitaliste de développement, à parler des « externalités négatives » induites et à prôner une "simplicité volontaire " pour infléchir le développement "sauvage » et on peut également rappeler la naissance de l'écologie dans ce même élan de prise de conscience des préoccupations environnementales avec l'américain Marsh G. P. (« Man and Nature ", 1864) ou l'allemand Haeckel E. (1866 avec la notion même d'écologie).

On peut donc légitimement s'interroger sur l'impact des modifications que les environnements, les paysages, la biodiversité ont pu subir durant la deuxième moitié 
du XIXe siècle (Chansigaud, 2012) et sur la perception qu'en ont eue des artistes citoyens. Et si le paysage impressionniste devenait lui-même une véritable prise de conscience de la gravité de ces modifications, la métaphore d'un acte citoyen, celui de dénoncer, consciemment ou inconsciemment, les atteintes environnementales? Et si, en tentant de peindre la fugacité des choses, la mobilité des nuages, le souffle du vent dans les branches, la variabilité de la lumière, ces artistes voulaient montrer l'instabilité d'un monde devenu trop rapide?

\section{Des « pré-impressionnistes " aux œuvres " réalistes ", en passant par Impression, soleil levant de C. Monet. ...}

5 Considérés comme "pré-impressionnistes ", annonçant à différents titres le courant qui allait prendre tant d'ampleur par la suite, Turner (1775-1851), Corot (1796-1875), Jongkind (1819-1891) et Boudin (1824-1898) ont peint des paysages dans lesquels les ciels, les conditions atmosphériques, les phénomènes météorologiques prennent une importance indéniable. Pour autant, on peut sans grande erreur montrer que leurs recherches picturales respectives ne relèvent pas du même parti pris. Autant Corot, Jongkind et Boudin s'attachent à montrer les conditions météorologiques telles qu'ils les perçoivent visuellement et telles qu'elles sont naturellement: cumulus de beau temps, tempête, vent dans les arbres... autant Turner réinterprète dans ses œuvres les cieux qu'il peint. Peu nombreuses sont, dans la totalité de l'œuvre peinte du Britannique, les toiles qui ne relèveraient pas d'une réinterprétation de ses propres sensations visuelles. C'est un argument non négligeable pour dénoncer le rapport proposé par certains auteurs entre l'éruption du Tambora en 1815 et différentes toiles (notamment «Didon construisant Carthage », 1815) datant de la même année ou des années immédiatement postérieures. Si l'on ne peut exclure que Turner ait observé, à certaines occasions très ponctuelles dans le temps, des cieux fortement influencés par la diffusion dans l'atmosphère de particules volcaniques ayant eu un impact indéniable sur la couleur du ciel, il faudrait envisager, à analyser sa production le long de sa vie, que les cieux de toute la première moitié du XIX ${ }^{e}$ siècle en Angleterre aient été bien différents de ceux observés par les peintres continentaux, en permanence marqués par la réfraction des ondes lumineuses sur les poussières volcaniques (sans compter que les éruptions sur le globe semblent avoir été beaucoup plus nombreuses dans la seconde moitié du XIX siècle, ce qui devrait donc inciter à beaucoup de prudence interprétative)!

6 À l'opposé pictural et chronologique de ces tableaux pré-impressionnistes et à la «fraicheur» de leurs toiles, des œuvres comme celles de Constantin Meunier (1831-1905) ou du jeune Paul Delvaux (1897-1994) paraissent parfaitement illustrer l'impact de quelques décennies de révolution industrielle sur l'œil d'un artiste, à la fois par leurs conséquences sociales, mais aussi environnementales.

7 D'une façon engagée picturalement et politiquement, le peintre belge Constantin Meunier s'inscrit, surtout à partir de 1878 , dans une vision du monde ouvrier dans laquelle le "pays noir » de la sidérurgie domine. Le nombre de toiles aux cheminées imposantes et actives (jusqu'à une dizaine dans certaines toiles des années 1890) est tel que le ciel s'obscurcit presque totalement, traduisant les sombres transformations que le monde rural de la province du Hainaut a subies en l'espace de quelques décennies. 
Avec ses paysages industriels et ferroviaires, comme «Fours à chaux sur la route d'Engis » $\left(1923^{3}\right)$, le jeune Paul Delvaux - il a alors 26 ans - peint des univers sombres, noirs de fumée, des « atmosphères » irrespirables, qui certes correspondent sans doute à ses états d'âme, mais dans lesquels l'activité industrielle est plus que pesante. Le noir domine, le "climat » est proche de celui de Germinal, les rails conduisent à un monde peu engageant où le vert de la végétation s'assombrit, devenant quasiment noir. Mais il est vrai aussi que l'on est là quelques années après la Première Guerre mondiale, dans une période également bien sombre par de nombreux aspects.

Un demi-siècle de transformations majeures donc, durant lequel s'inscrit la plus grosse partie de la production artistique de Claude Monet, né en 1840, mort en 1926, et qui, avec «Impression, Soleil levant » (figure 1), donne naissance, au demeurant sans le vouloir, au mouvement impressionniste. Cette toile n'est effectivement pas anodine puisque c'est à l'occasion de sa première exposition en 1874 - elle aurait été peinte un peu avant, durant l'hiver $72-73^{4}$ - que le mot même d'Impressionnisme est lancé, d'abord de manière péjorative puis en donnant un sens finalement assez cohérent à ce mouvement naissant. Mais elle n'est pas anodine, à plus d'un titre, car avec cette toile devenue fondatrice Monet exprime pleinement une «impression » qui ne relève pas, comme c'est le cas pour les œuvres antérieures de Corot ou de Boudin, d'une atmosphère, d'une impression seulement «naturelle ", et ce n'est pas non plus, a priori, une interprétation personnelle comme aurait pu le faire Turner. «Jusqu'à cette date, Monet n'a jamais peint Le Havre que sous l'aspect séduisant d'une station balnéaire. Marqué par son voyage à Londres, par le spectacle de la Tamise, du fog et des fumées d'usines, tout autant que par l'exemple de Turner, il peint pour la première fois la face industrielle et laborieuse du Havre, son port dans la brume » (Mathieu, 2017).

Figure 1. Impression soleil levant

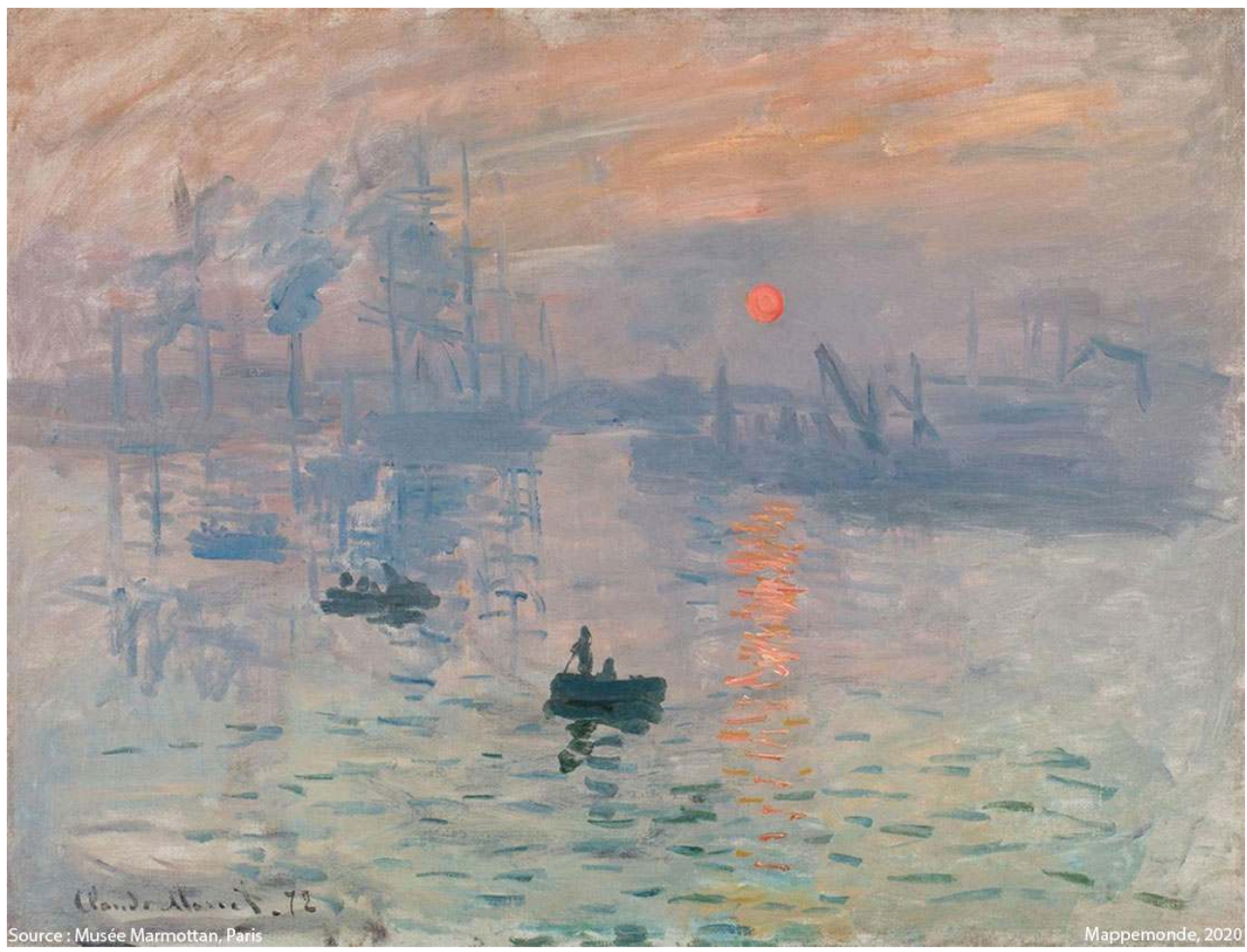

Monet, 1872, Musée Marmottan, Paris 
10 cheminées de navires à vapeur ${ }^{6}$ émettent leurs panaches de fumée (dans la partie gauche de la toile). Si ces fumées créent une ligne directrice symétrique à celle des trois barques $^{7}$, composant ainsi un point de fuite à l'extrême gauche du tableau - ce qui relèverait peut-être d'un effet voulu par Monet, elles semblent alimenter le halo qui masque partiellement le soleil levant. Monet peint un ciel pollué par l'activité industrielle et par la marine marchande du port du Havre. Et la trajectoire supposée du soleil au moment de sa réalisation (Olson in Mathieu et Lobstein, 2014) est parallèle à la directrice des fumées, ce qui fut peut-être pour Monet une sollicitation naturelle (la trajectoire du soleil du matin) pour renforcer à la fois son point de fuite vers la gauche et l'effet d'alimentation par ces mêmes fumées du halo qui cache le soleil. On peut effectivement et légitimement penser que les basses couches de l'atmosphère peinte par Monet sont dominées par la pollution (4, 5 et 6 de la figure 2), quand le haut de l'œuvre (un ciel plus « pur»?) présente des couleurs plus chaudes.

Figure 2. Interprétation de l'œuvre

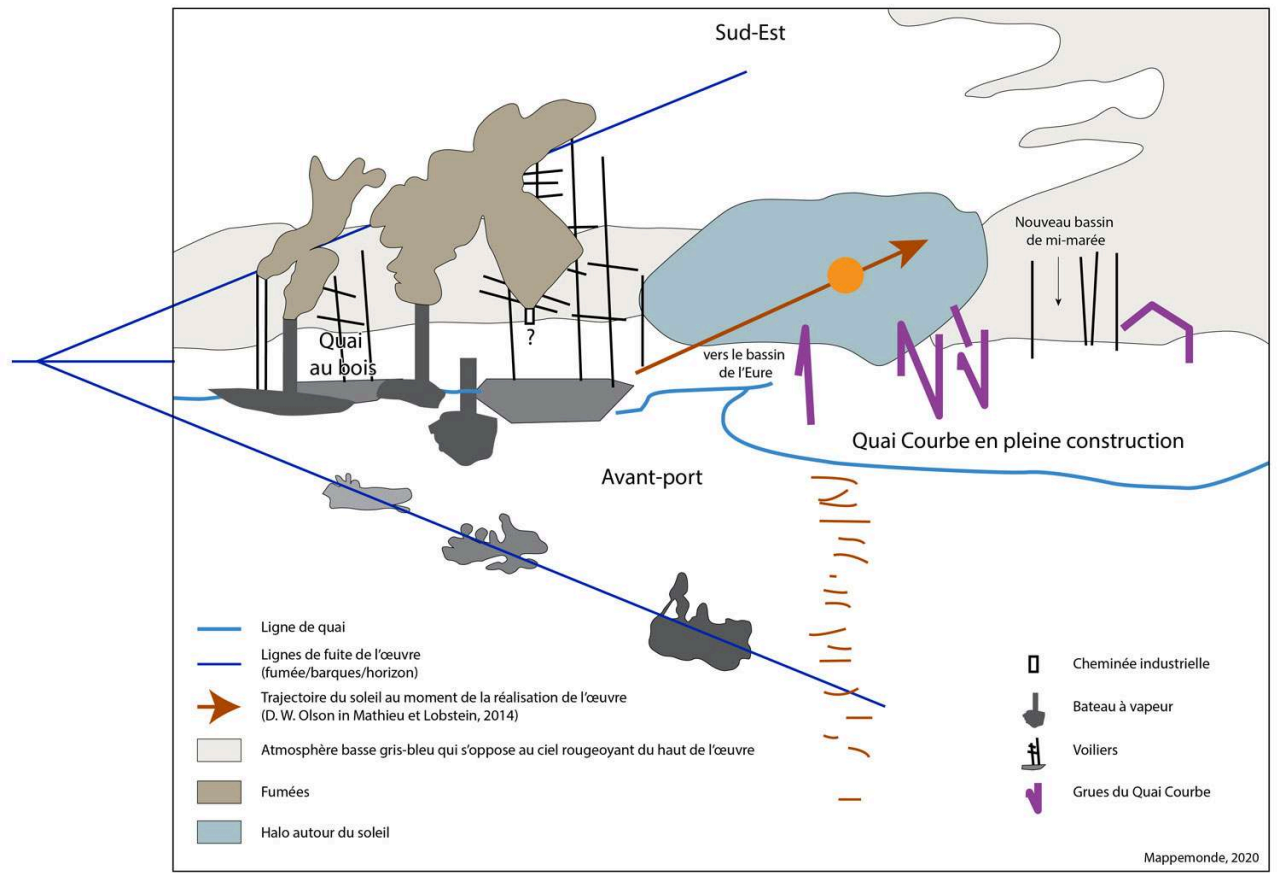

Les grues du Quai Courbe en pleine restructuration en 1872 afin de favoriser l'entrée des bateaux dans le port; derrière les grues, il ne peut s'agir que des premiers voiliers mouillant dans le tout nouveau bassin de mi-marée. Un doute subsiste quant à l'origine du panache de fumée de droite ; il semble bien émis par une cheminée, mais celle-ci reste très discrète, impossible à identifier !

Monet a-t-il seulement "joué avec la lumière » comme il l'a fait tout le long de sa carrière ? Ou a-t-il peint consciemment la pollution du port du Havre ? Rien ne permet de supposer que cette dernière idée ait été volontaire, pour autant n'est-on pas en droit de l'envisager? 


\section{Le Havre, « porte Océane » et port(e) d'entrée dans l'Anthropocène?}

13 Pour un historien de l'art, nul doute que l'argumentaire développé ci-dessus est sans aucun doute un peu « léger ", même si, comme les objets anciens qui nous interrogent davantage qu'ils nous dévoilent leurs significations profondes (Mac Gregor, 2018), les toiles peintes au XIXe siècle par des artistes qui ne nous ont pas livré toutes leurs motivations ou leurs interrogations artistiques profondes peuvent être perpétuellement reconsidérées, ce que font d'ailleurs tous les historiens de l'art depuis toujours. Mais si le paysage impressionniste est une "peinture de géographie", laissons aux géographes la possibilité de relire ce paysage, de tisser quelques liens entre œuvres picturales et «motivations citoyennes ». Comme le souligne si bien Thomson R. (in Alphant et al., 2011): "Je ne suis pas convaincu que notre étude de l'impressionnisme se soit intéressée aux préoccupations relevant de la géographie culturelle et de l'environnement (...). Il serait intéressant de voir de telles questions occuper le devant de la scène dans les recherches sur le paysage impressionniste ».

Postulons donc, à défaut d'avoir la certitude que Monet ait été le premier à penser l'entrée dans une ère nouvelle, dans une géographie nouvelle, que son tableau «Impression, soleil levant » le fit pour lui en 1874 lors de sa première exposition au public $^{8}$. Dans une ère nouvelle de la peinture avec le développement de l'impressionnisme évidemment, mais aussi pour nous dans cette relecture environnementale du XIX ${ }^{e}$ siècle, dans l'Anthropocène ${ }^{9}$.

Monet exprime avec ce tableau, sans doute pour la première fois, ce type d'atmosphère, il n'est probablement pas le seul ni le premier à vouloir peindre la brume ou le brouillard. Whistler (1834-1903) et Daubigny (1817-1878) l'ont également tenté (Corbeau-Parsons, 2018), mais Monet est celui qui semble vouloir montrer l'impact de la pollution des machines à vapeur dans l'atmosphère, les probables conditions anticycloniques ne permettant pas aux panaches de disparaître rapidement. C'est l'effet «pollution » de nos villes actuelles, surplombées par une nappe colorée peu engageante (et l'accentuation de la couleur chaude du soleil ?), par temps anticyclonique chaud ou froid, les polluants restant dans les basses couches de l'atmosphère, rendant l'air irrespirable (et favorisant la stagnation du halo de l'œuvre ${ }^{10}$ ?). Les peintures antérieures de Boudin, ou de Monet lui-même (par ex. « Jardin à St Adresse ", 1866-67) exploitaient déjà picturalement ces panaches de fumée industrialo-portuaires, mais c'était uniquement pour représenter une simple réalité (les bateaux dans le port), sans mettre en évidence la relation fumées/pollution atmosphérique. Or, lors de son premier séjour à Londres (en 1870, fuyant la guerre franco-prussienne), Monet a pu être sensibilisé au brouillard récurrent de Londres, mais aussi à toutes les conditions sanitaires de la ville. La prise de conscience d'une modernité engendrant pollutions et risques sanitaires graves a fortement marqué les Britanniques, notamment à la suite de "La Grande Puanteur » ("The Great Stink ») de 1858, et on peut envisager que Monet ait été sensible à ces problèmes urbains.

Assez paradoxalement donc, « Impression, soleil levant » relève davantage, alors que la toile a donné son nom au mouvement impressionniste, de l'esquisse réaliste. Esquisse parce que la liberté de la touche est plus proche d'un "instantané », d'une "vitalité » que l'on veut éloignés de la peinture académique, et réaliste parce que, à l'instar de " $\mathrm{La}$ petite danseuse de quatorze ans » modelée par Degas quelques années plus tard (vers la 
fin de la décennie 1870), il pourrait s'agir pour Monet « de susciter un étonnement, un choc salutaire qui ouvrent la conscience du spectateur en lui présentant non pas une œuvre élégante destinée à flatter son goût esthétique, mais le drame d'une société auquel il contribue » (Laurens, 2018) ${ }^{11}$. Sous cet angle de vue, il n'est pas surprenant que ces œuvres, considérées toutes deux comme inachevées, aient suscité respectivement autant de réactions lors de leur première exposition. Pour Degas, sa danseuse « dépourvue de tout effet destiné à embellir le réel, comme les romans de Zola ou de Maupassant, heurte le goût bourgeois ", décrit davantage "l'horreur (du) destin social et intime » (Laurens, 2018) des petits rats de l'Opéra vivant dans la pauvreté, dans les quartiers sordides, se prostituant même parfois pour survivre. Pour Monet, l'aspect séduisant des plages normandes de ses toiles antérieures (comme de celles de Boudin) est totalement oublié, la présence de l'homme, en tant qu'individu profitant des bienfaits d'une nature accueillante, d'un paysage immuable, étant supplantée par les impacts d'une société « capitaliste sauvage » qui transforme rapidement leur cadre de vie. L'homme est « oublié », l'homme est « exclu » du paysage impressionniste, comme le remarque si justement Baudelaire en regardant les pastels de Boudin dans son atelier (Alphant et al., 2011). L'homme exclu, mais une « outrance » apparente (« couleurs trop intenses, valeurs trop voisines, falaise trop grosse, cathédrale trop proche, nymphéas en série, partout l'excès "; ibidem) qui pourrait aussi signifier, symboliser l'outrance des modifications subies par les environnements.

Mais pourquoi Monet, qui a pu être sensible à son retour de Londres à la désormais trop fréquente pollution urbaine ou industrialo-portuaire, n'inscrit-il pas toute sa carrière postérieure dans cette veine réaliste ? Dans une possible dénonciation citoyenne de la dégradation des paysages et des environnements?

Les paysagistes impressionnistes ne sont pas urbains, ils ne sont pas parisiens. Le paysage impressionniste est « d'abord un art du paysage rural, avec comme berceaux ceux de l'estuaire de la Seine et de la Manche » (Dunet, 1975; on pourrait ajouter à cette citation tout le bassin versant de la Seine dans un large rayon autour de Paris). N'y aurait-il pas, chez les impressionnistes, le souci de mettre en valeur la main de l'homme dans les paysages ruraux depuis des millénaires, dans ces paysages ruraux qui évoluent désormais très vite, plus ou moins abandonnés pour certains par l'exode rural et agricole? Même dans la vallée de la Seine, les versants pentus jadis exploités (en vergers, vignobles, pâturages...) sont progressivement délaissés, désormais longés par ce chemin de fer Paris-Rouen-Le Havre si souvent représenté. Les impressionnistes seraient-ils donc à regretter un temps passé où les paysages, façonnés lentement par les générations antérieures, restaient (ou semblaient) immuables? Ils sont provinciaux, ils " sont Japonais, ils sont XVIII siècle " (Alphant M. in Alphant et al., 2011; de Butler, 2004). Le paradis pour peintre n'est-il pas «la sauvage et saisissante majesté d'une nature vierge de toute trace moderne, entremêlée partout de ruines druidiques, religieuses ou féodales qui s'y trouvent comme les pages éparses d'une histoire oubliée » comme le dit l'écrivain britannique Thomas Troloppe dès 1837 à propos de Pont-Aven vite devenu «repaire international de peintres»? (In "A summer in Brittany ", publié en 1840 ; Fémélat, 2017). Ces derniers seraient-ils alors sensibles à une autre forme de relation au paysage, à l'instar de celle des Japonais, sensibles aux codes esthétiques du jardin, de la nature idéalisée par la main de l'Homme? N'est-ce pas vers cette forme d'esthétique que se sont orientés Monet de 1883 à sa mort à Giverny, avec son jardin, ses nymphéas, son pont "japonais", ou Caillebotte (1848-1894) dans le domaine familial d'Yerres, tous deux correspondant et visitant le 
jardin de l'autre ? Est-ce par goût de la pensée orientale ou par un « repli sur soi » que les transformations des paysages ruraux suscitaient (un peu comme aujourd'hui le « retour au jardin » d'urbains totalement déconnectés de leurs racines rurales) ? Est-ce pour cela que le paysage impressionniste régional (dont la vitalité n'est plus à démontrer avec les "écoles» de Rouen, de Nancy, de Crozant, de Barbizon...) est aujourd'hui davantage signifiant, expliquant par là même le développement récent des expositions qui lui sont consacrées (« Les maîtres de la Creuse » en 1997, les expositions de Nancy en 1999 et de Rouen depuis 2010...; Thomson in Alphant et al., 2011).

Les paysagistes de l'impressionnisme, souvent d'origines rurales, ont-ils préféré l'esthétisme d'environnements encore peu transformés par la révolution industrielle, de paysages ruraux encore fortement marqués par les activités traditionnelles (cf. «la série des meules») et se sont-ils, dans une forme de refus inconscient, évertués à montrer une nature "idéalisée ", sans cesse reconstruite, remodelée par la main de l'homme, sans cesse "jardinée", pour n'y voir que "les effets de la lumière sur le beau $»^{12}$ ?

Monet, avec "Impression, soleil levant » et après son voyage à Londres en 1870, a-t-il voulu dénoncer la pollution importante du port du Havre ? La question pourra toujours rester sans réponse vraiment convaincante.

21 Mais, à une époque où le paysage, après avoir quasiment disparu comme objet d'étude du géographe, revient en force dans les préoccupations liées à la mise en valeur des aménités environnementales, à la patrimonialisation... (Qu'on en juge en termes de textes législatifs... !), à une époque où une réelle "perte de valeurs » est ressentie par bon nombre des citoyens (qui n'est pas sans rappeler le souci identitaire et patriotique du début du XX $\mathrm{X}^{\mathrm{e}}$ siècle et son triptyque pays/paysage/paysans), et à une époque où l'on se demande quand l'Humanité est entrée dans l'Anthropocène, on peut envisager l'idée que la toile de Monet de 1872 en est la première manifestation picturale.

Et donc à la question (certes saugrenue) «par où entre-t-on dans l'Anthropocène? », gageons que ce soit par le port du Havre et sa porte Océane!

\section{BIBLIOGRAPHIE}

ALPHANT M., CLAYSON H., THOMSON R., DOMBROWSKI A. (2011). « Impressionnisme(s) aujourd'hui ». Perspective, $\mathrm{n}^{\circ}$ 1/2011, p. 509-522. En ligne : https://journals.openedition.org/perspective/1016 BONNEUIL C., FRESSOZ J.B. (2016). L'événement Anthropocène. La Terre, l'histoire et nous. Paris : Seuil, 320 p. CHANSIGAUd V. (2012). Des hommes et des oiseaux : une histoire de la protection des oiseaux. Paris : Delachaux et Niestlé, 224 p. CORBEAU-PARSONS C., dir. (2018). Les impressionnistes à Londres. Artistes français en exil 1870-1904. Paris : Paris Musées, 271 p. 
D'ARCY WOOD G. (2019). L'année sans été, Tambora 1816, le volcan qui a changé le cours de l'histoire. Paris : La Découverte, $336 \mathrm{p}$.

DE BUTLER A. (2004). Lumières sur les impressionnistes. Ironie, $\mathrm{n}^{\circ}$ 102. En ligne : http://ironie.free.fr/ iro_102.html

DUNET M. (1975). Flaubert, artiste et précurseur de l'impressionnisme. Les amis de Flaubert, $\mathrm{n}^{\circ} 46$.

FEMELAT A. (2017). Gauguin. D'art et de Liberté. Neuilly-sur-Seine : Michel Lafon/ Issy-les-

Moulineaux : Beaux-arts Éditions. $224 \mathrm{p}$.

LAURENS C. (2018). La petite danseuse de Degas. Paris : Gallimard. 173 p.

MATHIEU M., LOBSTEIN D., dir. (2014). Impression, soleil levant. L'histoire vraie du chef-d'œuvre de Claude Monet. Paris : Hazan/Musée Marmottan Monet. 223 p.

MATHIEU M. (2017). «Impression, soleil levant : paradigme de l'impressionnisme ou anti-modèle ?» In Impression(s), soleil, Paris : Somogy Éditions d'art/Le Havre : MuMa, p. 117-130.

MAC GREGOR N. (2018). Le monde en cent objets. Paris : Les Belles Lettres, 862 p.

METZGER A., TABEAUD M. (2010). Les scènes hivernales dans la peinture du Siècle d'or hollandais. Physio-Géo - Géographie Physique et Environnement, vol. 4-2010. En ligne : https://journals.openedition.org/ physio-geo/1312?lang=en

METZGER A., DESARTHE J. (2017). Regarde s'il pleut : Effets d'inondations dans la peinture française

(1856-1910). Communications, $\mathrm{n}^{\circ}$ 101, p. 119-141.

THORNES J. E., METHERELL G. (2003). Monet's 'London Series' and the cultural climate of London at the turn of the Twentieth Century. In S. STRAUSS et B. S.ORLOVE, éd. Weather, climate and culture, Oxford : Berg Publishers. p. 141-160.

ZEREFOS C.S., TETSIS P., KAZANTZIDIS A., AMIRIDIS V., ZEREFOS S. C., LUTERBACHER J., ELEFTHERATOS K., GERASOPOULOS E., KAZADZIS S., PAPAYANNIS A. (2014). Further evidence of important environmental information content in red-to-green ratios as depicted in paintings by great masters, Atmos.Chem.Phys, 14, p. 2987-3015.

\section{NOTES}

1. Géographe et géomorphologue, Jean-Jacques Dufaure était un enseignant humaniste, amoureux de la Grèce, de l'Italie (où il a dirigé mes recherches de thèse en Ombrie et dans les Marches) et du monde méditerranéen. Il était aussi très intéressé par le monde de l'archéologie et par celui de l'art en général. C'est à ce titre que je lui dédie cet article qui est le dernier texte que je lui avais envoyé avant soumission à Mappemonde. Il n'a pas eu le temps ou la force de me retourner ses habituelles et judicieuses remarques.

2. "Procédé qui consiste à faire reconnaître une image connue dans une image nouvelle ». https://fr.wiktionary.org/wiki/intericonicité

3. La Gazette Drouot, 2018, 41.

4. Elle est signée « 72 », mais différentes propositions de dates de réalisation ont été soumises, en tenant compte des conditions atmosphériques et des marées (D. W. Olson in Mathieu et Lobstein, 2014).

5. C'est au moins une, peut-être deux cheminées, qui se remarque(nt) sur ce qui serait le Quai au Bois (ou quai de Broström); il s'agit de cheminées qui actionnaient les appareils hydrauliques pour vider l'eau des cales (pour mettre les navires à sec), dépendantes du Bassin de la Citadelle 
(inaugurées en 1871). Sur la photo publiée par G. Lefebvre, on voit bien deux cheminées sur ce quai (in Mathieu et Lobstein, 2014 ; fig. 6, p. 63.)

6. En 1872, le port du Havre reçoit 1182 navires à vapeur (569 en 1860) et encore 1183 bâtiments à voiles (contre 1864 en 1860). Les remorqueurs qui amenaient les voiliers dans les différents bassins étaient à vapeur et à aubes. Sur l'œuvre, on en remarque un qui semble remorquer le grand voilier à l'arrière-plan (derrière la petite barque centrale).

7. S'agit-il de trois barques ou de la même en progression dans le temps (G. Lefebvre in Mathieu et Lobstein, 2014) ? Elles ont en tout cas été peintes à la fin du travail de Monet, comme le soleil et ses reflets dans l'eau (Ibid.).

8. Trois autres tableaux de Monet pourraient être associés à notre réflexion : le "Soleil levant » conservé au J.-P. Getty Museum de Los Angeles, peint sans doute le même matin et qui pourrait être « décortiqué » picturalement presque de la même façon, ainsi que «Bateaux dans le port de Londres » (signé « Claude Monet - London ») et « Le port de Londres » (conservé à Cardiff) peints lors de son voyage à Londres durant la guerre franco-prussienne, dans lesquels cheminées d'usine et de bateaux à vapeur sont bien présentes.

9. Rappelons que le début de la période «Anthropocène " (période pendant laquelle les activités humaines auraient marqué la Terre de leur empreinte) est toujours en pleine discussion auprès de l'UIGS (Union Internationale des Sciences Géologiques) : extinction des grandes faunes du Pléistocène supérieur? Début de l'agriculture et de la riziculture $\left(\mathrm{VIII}^{\mathrm{e}}\right.$ et $\mathrm{V}^{\mathrm{e}}$ millénaires avant J.C.) ? Découverte de l'Amérique en 1492? Révolution industrielle ?... Dans notre propos, ce seraient les grands changements sociétaux et environnementaux liés à la révolution industrielle en Europe qui s'exprimeraient dans la peinture impressionniste.

10. C'est un des aspects qui n'a, à notre connaissance, jamais été vraiment discuté : halo brumeux anticyclonique ? ou halo de pollution ? Stendhal en 1838 décrit la pollution du port du Havre qu'il compare à celle de Londres; en 1872 l'activité portuaire était encore plus intense (D.W. Olson in Mathieu et Lobstein, 2014). Les dates avancées pour la réalisation de cette toile ont été listées en fonction des jours possibles de brume et de brouillard, mais quid des jours anticycloniques favorables à une stagnation des polluants dans les couches basses de l'atmosphère ? (Ibid.).

11. Certes, Monet ne semble pas avoir dit expressément dans ses commentaires avoir voulu peindre cette pollution...

12. Metzger A. et Desarthe J. (2017) montrent que les impressionnistes idéalisent aussi d'une certaine façon les inondations, ne montrant ni dégâts, ni victimes... contrairement aux artistes antérieurs.

\section{RÉSUMÉS}

L'œuvre de Monet «Impression soleil levant », datée de 1872, peut-elle être considérée comme la première manifestation picturale de l'entrée de l'Humanité dans l'Anthropocène? La question mérite d'être posée dans la mesure où le peintre paraît souligner davantage les effets de la pollution industrialo-portuaire que les simples effets de lumière d'un soleil levant dans la brume. La toile qui a donné naissance au courant de l'impressionnisme en marquant fortement les esprits lors de sa première exposition, au demeurant à l'instar de " la petite danseuse de Degas ", relève davantage de l'esquisse réaliste, peut-être d'une prise de conscience du citoyen Monet peu de temps après son voyage à Londres (1870). 
INDEX

Mots-clés : anthropocène, impressionnisme, Le Havre, Monet

\section{AUTEUR}

MICHEL RASSE

Université Lyon 2, UMR Archéorient 